\title{
Soil structure interaction for integral abutment bridge using spring analogy approach.
}

\begin{abstract}
The reaction of the backfill behind the abutments and adjacent to the piles plays a significant role in the behavior of the Integral bridge. The handling of soil-structure interaction in the analysis and design of integral abutment bridges has always been problematic due to its complexity. This study describes the implementation of a 2-D finite element model of IAB system which explicitly incorporates the soil response. The superstructure members and the pile have been represented by means of three-node isoperimetric beam elements with three degree of freedom per node. The Eight node isoperimetric quadrilateral element has been used to model the abutment. The backfill was idealized by uncoupled 'Winkler' spring. The appliclability of this model is demonstrated by analyzing a single span IA bridge. The results have shown that the shear forces at the tops of the supported piles were only $12 \%$ to $16 \%$ of the load which at the top of abutment.
\end{abstract}

Keyword: Analysis and design; Beam elements; Degree of freedom; Eight node; Finite element models 